\title{
The $\phi$ mean free path in hot hadronic matter
}

\author{
L. Alvarez-Ruso and V. Koch \\ Nuclear Science Division, Lawrence Berkeley National Laboratory \\ 1 Cyclotron Rd., Berkeley, CA 94720, USA
}

\begin{abstract}
We calculate the $\phi$ meson collision rate and mean free path in a hot hadronic gas. The Hidden Local Symmetry model is used to take into account interactions of $\phi$ mesons with pseudoscalar $(\pi, K)$ and vector mesons $\left(\rho, \omega, K^{*}\right.$, $\phi)$. In contrast to previous calculations we find a significantly small mean free path (around $1 \mathrm{fm}$ at $\mathrm{T}=200 \mathrm{MeV}$ ). This implies that $\phi$ mesons produced after hadronization in relativistic heavy ion collisions will not leave the collision region without scattering. The consequences of these findings to the analysis of $\phi$ yields are discussed.
\end{abstract}

PACS numbers: 25.75.-q, 14.40.Ev, 24.10.Pa, 12.39.Fe

The main motivation for studying $\phi$ production in relativistic heavy-ion collisions is related to the fact that the formation of quark-gluon plasma should lead to enhanced strangeness [1]. In this case, free $s \bar{s}$ pairs would coalesce to form $\phi$ mesons, while their production in $p p$ and $\pi p$ collisions is OZI suppressed [2]. On the other side, $\phi$ properties (mass, decay width) are expected to be modified due to both many body effects in the hadronic medium and chiral symmetry restoration.

It is widely accepted that $\phi$ mesons have a large mean free path (MFP) in hot hadronic matter. Available calculations [3, 4, 5] seem to support this idea. For example, in Reference [4] a rather big MFP of $\lambda=4.4 \mathrm{fm}$ at $T=200 \mathrm{MeV}$ and $15 \mathrm{fm}$ at $T=150 \mathrm{MeV}$ was obtained. These calculations consider only a limited number of mechanisms, using mainly couplings that could be extracted from experimental partial decay rates (like $\rho \rightarrow \pi \pi$ or $K^{*} \rightarrow K \pi$ ). However, there are many vertices ( $\phi K^{*} K$, $\rho K^{*} K^{*}$, etc.), which could be relevant for the study of $\phi$ propagation in a hadronic medium, allowed by the symmetries of QCD but whose couplings cannot be extracted directly from experiment.

Therefore, it is important to tackle this problem in a framework where at least the low-lying pseudoscalar and vector mesons are treated in a manner consistent with the symmetries of QCD. One such a model is the so called Hidden Local Symmetry (HLS) Lagrangian developed by Bando and coworkers [6]. It is based on the fact that a $\left[\mathrm{U}(3)_{\mathrm{L}} \times \mathrm{U}(3)_{\mathrm{R}}\right] / \mathrm{U}(3)_{\mathrm{V}}$ non-linear sigma model is gauge equivalent to another one with $\left[\mathrm{U}(3)_{\mathrm{L}} \times \mathrm{U}(3)_{\mathrm{R}}\right]_{\text {global }} \times\left[\mathrm{U}(3)_{\mathrm{V}}\right]_{\text {local }}$ symmetry. Vector mesons stand for the gauge bosons of the hidden local $\mathrm{U}(3)_{\mathrm{V}}$ symmetry and acquire mass

$$
m_{V}^{2}=2 g^{2} f_{\pi}^{2}
$$

by spontaneous symmetry breaking. Meanwhile a Lagrangian describing the interaction of a vector meson with a pair of pseudoscalar ones (VPP) is also generated

$$
\mathcal{L}_{V P P}=-i g\left\langle\left\{\left[\Phi, \partial_{\mu} \Phi\right], V^{\mu}\right\}\right\rangle .
$$


$\Phi$ and $V_{\mu}$ are the octet of pseudoscalar Goldstone bosons and the nonet of vector mesons respectively; \langle\rangle denotes flavor trace. Notice that Equation 1 relates the VPP coupling to the vector meson mass.

Flavor symmetry breaking can be taken into account without affecting the HLS [7]. This leads to different vector meson masses and VPP couplings. For example,

$$
g_{\rho K K}=\frac{g}{2} \frac{1}{\left(1+c_{A}\right)}, \quad g_{K^{*} K \pi}=\frac{g}{2} \frac{\left(1+c_{V}\right)}{\sqrt{1+c_{A}}},
$$

where $g, c_{V}$, and $c_{A}$ are extracted from the experimental values of the masses and the meson decay constants. The accuracy of the model can be illustrated by comparing the decay widths $\Gamma\left(K^{*} \rightarrow K \pi\right)=51.5 \mathrm{MeV}$ and $\Gamma\left(\phi \rightarrow K^{+} K^{-}\right)=2.4 \mathrm{MeV}$ with the experimental values (50.8 and $2.17 \mathrm{MeV}$ respectively).

We also include the anomalous part of the Lagrangian [8] which contains the vector-vector-pseudoscalar interaction

$$
\mathcal{L}_{V V P}=2 g_{V V P} \epsilon^{\mu \nu \lambda \sigma}\left\langle\partial_{\mu} V_{\nu} \partial_{\lambda} V_{\sigma} \Phi\right\rangle,
$$

where $g_{V V P}$ is fixed by the anomaly. An important contribution is related to the fact that the kinetic term for the vector mesons contains self-interactions, which generate 3 and 4 vector meson vertices

$$
\begin{aligned}
& \mathcal{L}_{V V V}=i g\left\langle\left(\partial_{\mu} V_{\nu}-\partial_{\nu} V_{\mu}\right)\left[V^{\mu}, V^{\nu}\right]\right\rangle \\
& \mathcal{L}_{V V V V}=-\frac{g^{2}}{2}\left\langle\left[V_{\mu}, V_{\nu}\right]\left[V^{\mu}, V^{\nu}\right]\right\rangle .
\end{aligned}
$$

The VPP, VVP, VVV and VVVV interaction vertices can be combined to form Feynman diagrams for all possible binary reactions of $\phi$ 's with $\pi, K, \rho, \omega, K^{*}, \phi$ at tree level. The OZI rule manifests itself in the absence of direct couplings of $\phi$ 's with $\pi, \rho$

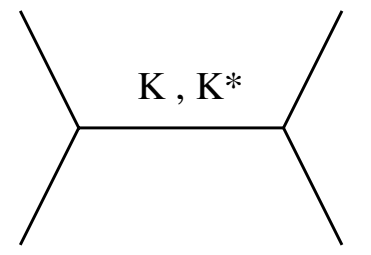

( s )

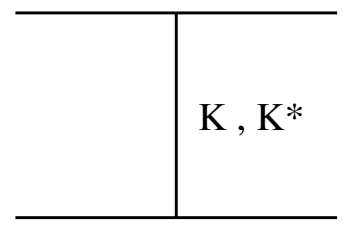

$(\mathrm{t})$

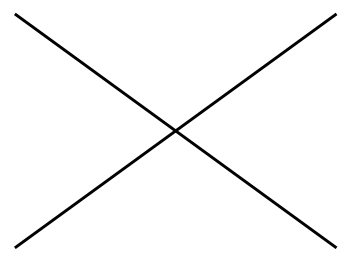

( c )

Figure 1. Generic Feynman diagrams for the different reaction mechanisms; (s) stands for s-channel, (t) for t-channel and (c) for contact.

or $\omega$ mesons in the model. Therefore, only s and t diagrams with a strange particle $\left(K\right.$ or $\left.K^{*}\right)$ in the intermediate state appear. This is also the reason why s-channel mechanisms are only present in the case of $\phi$ collisions with strange mesons.

We use standard expressions for the propagators

$$
\begin{aligned}
& D_{K}(q)=\frac{i}{q^{2}-m_{K}^{2}+i m_{K} \Gamma_{K}^{(t o t)}\left(q^{2}\right)}, \\
& D_{K^{*}}^{\mu \nu}(q)=-\left(g^{\mu \nu}-\frac{q^{\mu} q^{\nu}}{m_{K^{*}}^{2}}\right) \frac{i}{q^{2}-m_{K^{*}}^{2}+i m_{K^{*}} \Gamma_{K^{*}}^{(t o t)}\left(q^{2}\right)} .
\end{aligned}
$$


where $\Gamma_{K\left(K^{*}\right)}^{(t o t)}$ stand for the total decay widths of the intermediate $K, K^{*}$ and include all decay channels that are open for a given $q^{2}$ value. The presence of these imaginary parts implies an approximate unitarization of the s-channel diagrams. High momentum transfers in the t-channel diagrams are suppressed by the form factors

$$
F_{i}\left(q^{2}\right)=\frac{\Lambda^{2}-m_{i}^{2}}{\Lambda^{2}-q^{2}}, \quad i=K, K^{*}
$$

with $\Lambda=1.8 \mathrm{GeV}$, as chosen in Ref. [9] to reproduce kaon production cross sections. Finally, we have taken approximately into account the resummation of s-channel loops in the contact VVVV amplitude for $\phi+a \rightarrow b+c$ by means of the substitution

$$
\alpha g^{2} \rightarrow \frac{\alpha g^{2}}{\left[1+\alpha g^{2} G\left(s, m_{\phi}, m_{a}\right)\right]^{1 / 2}\left[1+\alpha g^{2} G\left(s, m_{b}, m_{c}\right)\right]^{1 / 2}},
$$

with

$$
G\left(s, m_{a}, m_{b}\right)=-i \frac{1}{2 \pi} \frac{p_{c m}}{\sqrt{s}}\left[1+\frac{\left(s-m_{a}^{2}-m_{b}^{2}\right)^{2}}{8 m_{a}^{2} m_{b}^{2}}\right],
$$

where $p_{c m}$ is the momentum of the vector mesons $a$ and $b$ in the CM frame. The factor $\alpha=-1 / 2$ for the $\phi \phi K^{*} K^{*}$ vertex and $\alpha=1 /(2 \sqrt{2})$ for $\phi \rho K^{*} K^{*}$ and $\phi \omega K^{*} K^{*}$. This ansatz is inspired by the solution of the Bethe-Salpeter equation in the K-matrix approximation using only the part of the tree-level amplitude, which leads to an algebraic equation.

With these ingredients one can compute the total cross sections and the collision rates i.e. the average number of collisions per unit time at a given temperature (in the zero width approximation for the initial and final states).

$$
\Gamma_{\text {coll }}^{(a)}=n_{a}\left\langle\sigma_{\phi a} v_{r e l}\right\rangle, \quad a=\pi, K, \rho, \omega, K^{*}, \phi .
$$

The contributions of different mesons to the $\phi$ collision rate as a function of temperature are shown in Figure 2.

In order to understand why $\Gamma_{\text {coll }}^{\left(K^{*}\right)}$ is bigger than $\Gamma_{\text {coll }}^{(K)}$ even if $K^{*}$ 's are considerably heavier than kaons, it is useful to look at the ratio of the density of states

$$
\frac{n_{K^{*}}}{n_{K}}=\left.\frac{3 m_{K^{*}}^{2} K_{2}\left(m_{K^{*}} / T\right)}{m_{K}^{2} K_{2}\left(m_{K} / T\right)}\right|_{T=200 \mathrm{MeV}}=0.77 .
$$

Its value at $T=200 \mathrm{MeV}$ is not as small as one would naively expect because the difference in masses and spin degeneracies almost compensate the ratio of Bessel functions. Therefore, to get a 1.7 times larger $\Gamma_{\text {coll }}^{\left(K^{*}\right)}$ it is enough to have a 2.3 times bigger cross section. The later is achieved due to the contribution of the reactions involving all four vector mesons, specially $\phi+K^{*} \rightarrow \rho+K^{*}$.

The total collision rate, obtained as the sum of the previous partial contributions, is shown in Figure 3. Inelastic ( $\phi$-number changing) reactions account for more than $80 \%$ of the total rate.

It is now straightforward to compute the mean free path as

$$
\lambda(T)=\frac{\bar{v}}{\Gamma_{\text {coll }}}
$$

with $\bar{v}$ being the average velocity of the $\phi$ in the medium. We have obtained a small mean free path: less than $2.4 \mathrm{fm}$ at temperatures above $170 \mathrm{MeV}$, as can be seen in Figure 4. Therefore, the $\phi$ mesons that are created after hadronization, at temperatures presumably between 170 and $200 \mathrm{MeV}$, will not leave the fireball without 


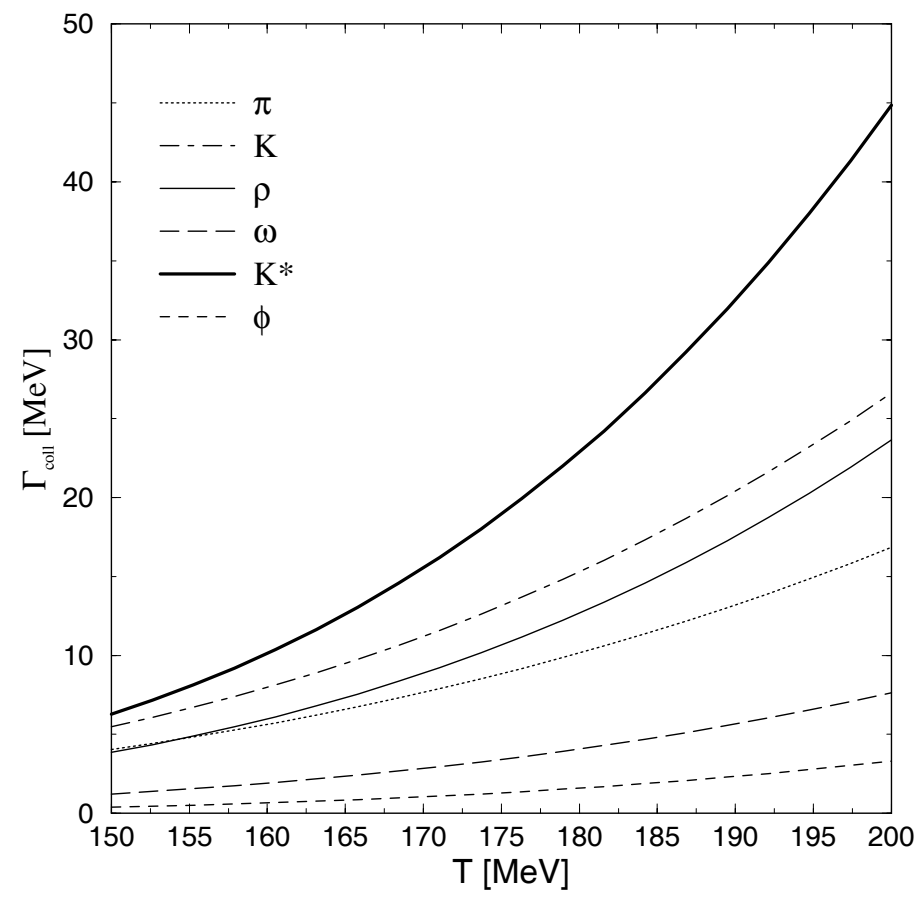

Figure 2. Collision rates of $\phi$ with $\pi, K, \rho, \omega, K^{*}$ and $\phi$ as a function of temperature.

interacting; they will rather remain in kinetic equilibrium at least during the early stage of the evolution in the hadronic phase. The uncertainties in the determination of the parameters in the HLS model and unitarization procedures are not likely to modify this conclusion.

Next, we have studied the implication of the previous result for the time evolution of the $\phi$ density in an expanding hadronic medium using a simple model. We have considered an ideal gas composed of $\pi, K, \rho, \omega, K^{*}$ and $\phi$, assuming kinetic equilibrium from hadronization to freezeout. For the sake of simplicity, chemical equilibrium has been assumed for all species except $\phi$ 's. In these conditions, the $\phi$ density is the solution of the following rate equation

$$
\partial_{\mu}\left(n_{\phi} u^{\mu}\right)=-\Gamma\left(n_{\phi}-n_{\phi}^{e q}\right),
$$

where

$$
n_{\phi}^{e q}(T)=\frac{3}{2 \pi^{2}} m_{\phi}^{2} T K_{2}\left(\frac{m_{\phi}}{T}\right),
$$

and $\Gamma(T)$ is the sum of the total collision rate calculated above and the averaged free decay width.

Then, we assume boost invariance and a uniform density distribution in the transverse plane and let the system expand both in the longitudinal and transverse radial directions. The ansatz for the radial flow velocity is inspired by hydrodynamics [10]

$$
\beta_{r}(\tau, r)=\beta_{s}\left(\frac{r}{R}\right) .
$$




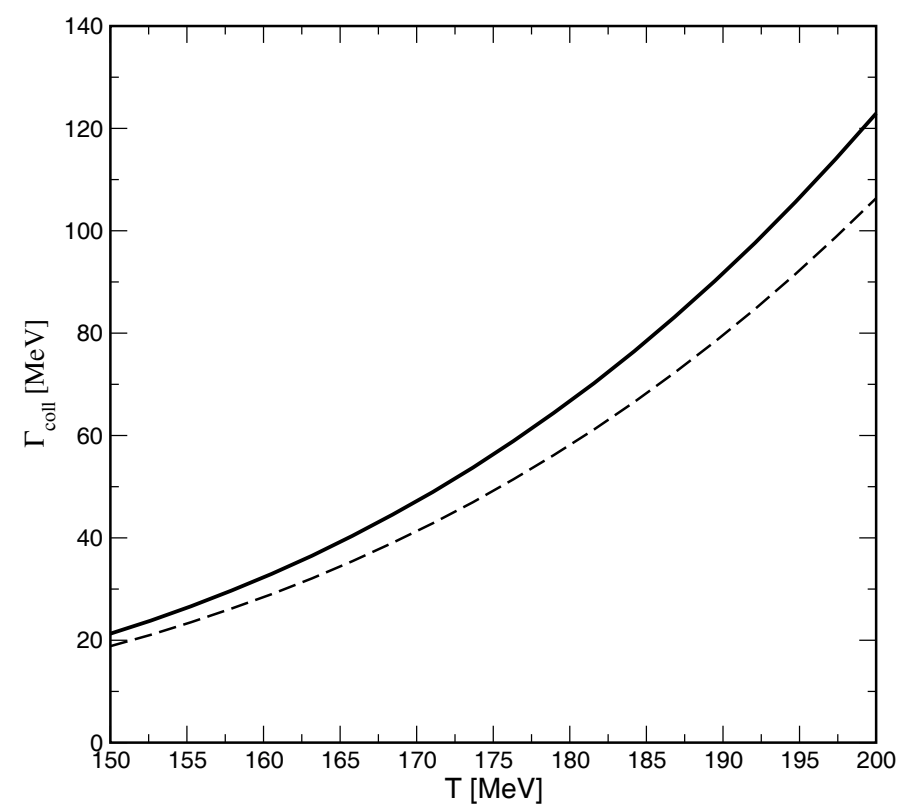

Figure 3. Total collision rate of $\phi$ meson as a function of temperature (solid line). The dashed line is the contribution from $\phi$-number changing processes alone.

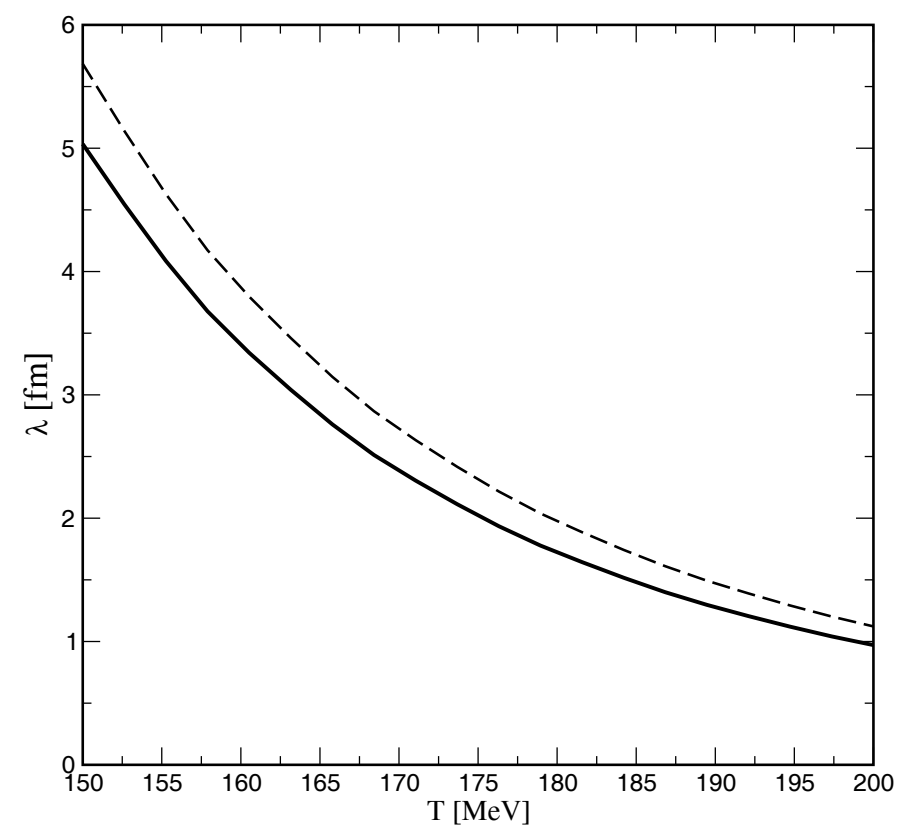

Figure 4. Temperature dependence of the $\phi$ mean free path in hot hadronic matter. Line styles have the same meaning as in Figure 3. 
In the case of a constant $\beta_{s}$, the transverse radius of the systems evolves as

$$
R(\tau)=\beta_{s}\left(\tau-\tau_{0}\right)+R_{0},
$$

where $R_{0}=1.2 A^{1 / 3} \mathrm{fm}$ is the radius of the colliding nuclei. The dependence of the proper time, which is nothing but the longitudinal size of the system at mid-rapidity, upon the temperature is dictated by entropy conservation

$$
\partial_{\mu}\left(s u^{\mu}\right)=0 .
$$

Notice that if $\Gamma=0$, Equation 15 implies $\phi$-number conservation throughout the expansion whereas if $\Gamma \rightarrow \infty$, then $n_{\phi}=n_{\phi}^{e q}(T)$ and the total number of $\phi$ 's will drop considerably. The actual solution (with our finite $\Gamma$ ) corresponds to an intermediate situation where the $\phi$-number decreases but not as fast as in the equilibrium case.

Figure 5 shows the $\tau$ dependence of the ratio of the $\phi$ yield at mid-rapidity $N(\tau)=$ $\tau \pi R^{2}(\tau) n_{\phi}(\tau)$ and the number of them right after hadronization $N_{0}=\tau_{0} \pi R_{0}^{2} n_{\phi}^{e q}\left(T_{0}\right)$. We have used $\tau_{0}=1 \mathrm{fm}, T_{0}=190 \mathrm{MeV}, \beta_{s}=0.6, a=1$ and $A=197(\mathrm{Au})$. At

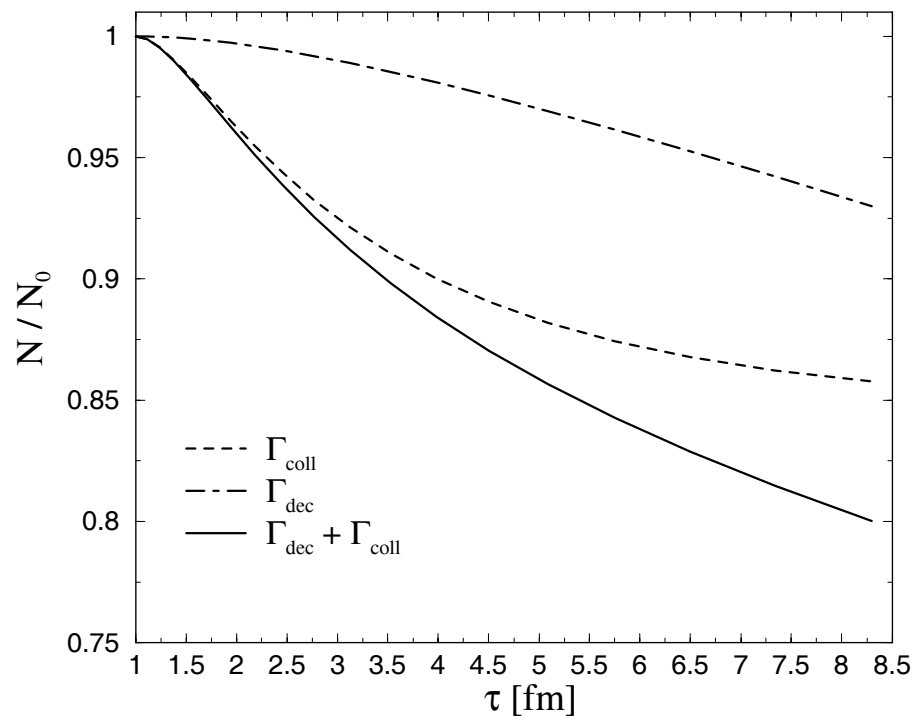

Figure 5. Time dependence of the ratio of the $\phi$ number to its value at hadronization. The dashed line shows the contribution from inelastic collisions while the long-short dashed one accounts for free decay (and recombination) The combined effect of both collisions and free decay is described by the solid line.

the early stages of the expansion, the system is hot and the collision rates are high, so that $\phi$ 's are pushed towards equilibrium making its number decrease fast. As the system cools down, the collision rates become small and their contribution to the ratio starts to saturate. We have taken a freezeout temperature of $T_{f}=100 \mathrm{MeV}$, which corresponds to a life time of $\tau_{f}=8.3 \mathrm{fm}$. If one assumes that only those $\phi$ mesons present at freezeout are detectable via kaon pairs, then the $\phi$ yield is reduced in $20 \%$ with respect to hadronization.

The dependence on the value of $\beta_{s}$ is illustrated in Fig 6 . The bigger $\beta_{s}$, the faster the temperature drops and, hence, the closer $N / N_{0}$ gets to one. We have also considered different hadronization temperatures, as shown in Fig. 7 . If the 


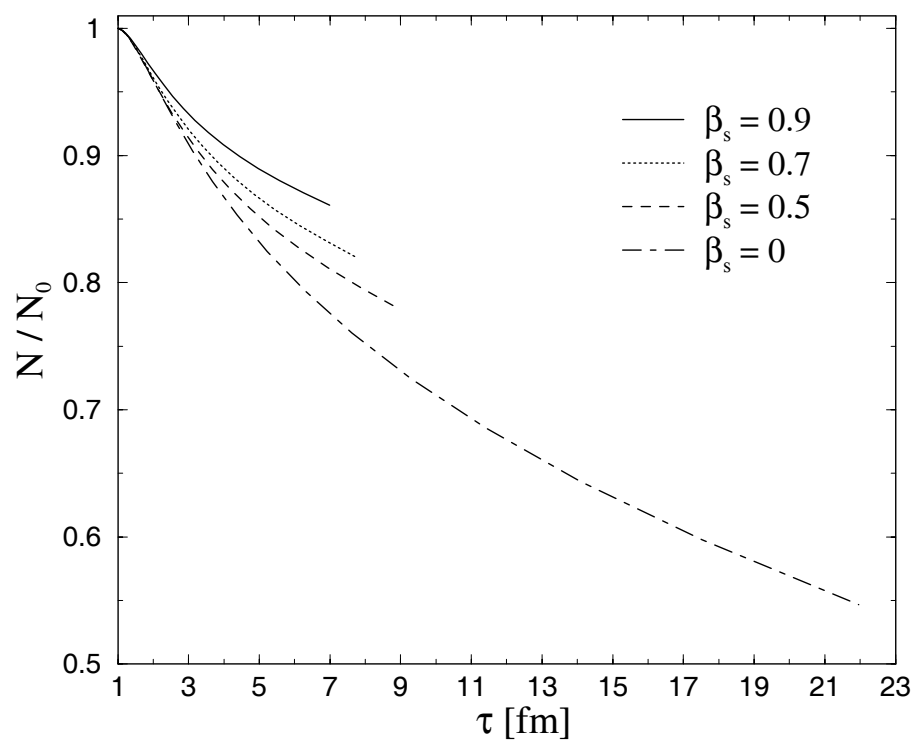

Figure 6. Ratio of the $\phi$ number to its value at hadronization for different values of the transverse flow velocity at the surface. The end point of the lines correspond to a temperature of $100 \mathrm{MeV}$.

hadronization temperature is low, the collision rates are small from the very beginning, and the interactions become less relevant.

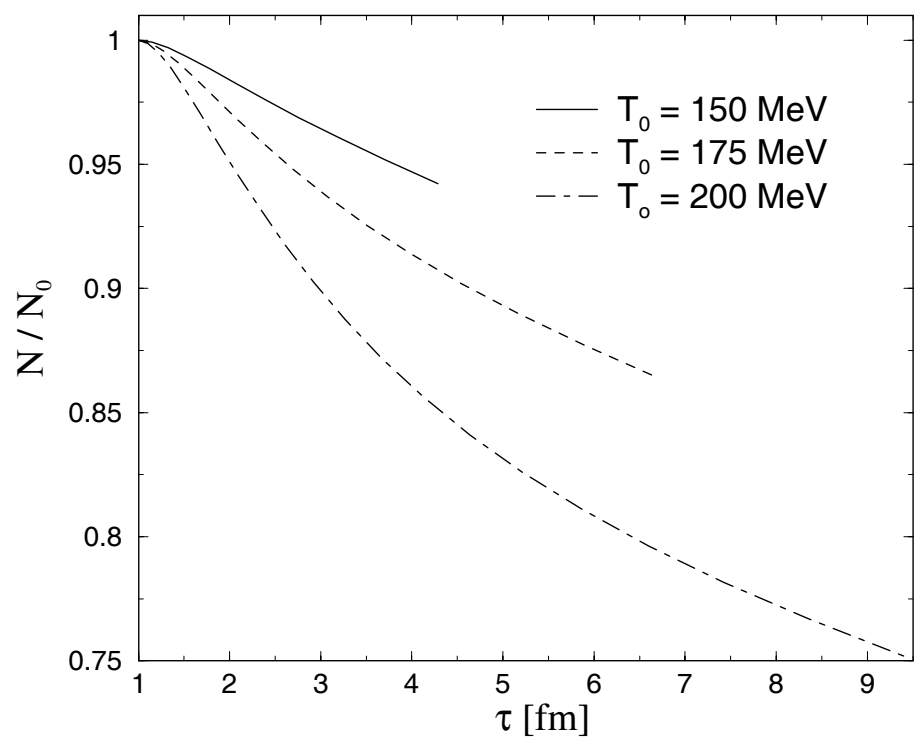

Figure 7. Same as Fig. 6 but for different hadronization temperatures. 


\section{Conclusions}

We have studied $\phi$ interactions with a hot hadronic medium composed of $\pi, K, \rho$, $\omega, K^{*}$ and $\phi$ using the Hidden Local Symmetry model. In this way, we could take into account many vertices that are allowed by the symmetries of strong interactions but whose couplings cannot be directly determined experimentally. As a consequence, at temperatures above $170 \mathrm{MeV}$, the $\phi$ mean free path is less than $2.4 \mathrm{fm}$ i.e. much smaller than the typical size of the hadronic system created in relativistic heavy ion collisions.

The implications of this result for the $\phi$ yield has been investigated by solving the rate equation for the $\phi$ density assuming kinetic but not chemical equilibrium. The high collision rates at the early stages of the hadronic evolution tend to maintain the equilibrium, causing a reduction of the $\phi$ number with respect to hadronization. This decrease ranges from $5 \%$ to $45 \%$ depending on hadronization temperature, freezeout temperature and flow velocity.

\section{Acknowledgments}

This work was supported by the Director, Office of Science, Office of High Energy and Nuclear Physics, Division of Nuclear Physics, and by the Office of Basic Energy Sciences, Division of Nuclear Sciences, of the U.S. Department of Energy under Contract No. DE-AC03-76SF00098. L.A.R. acknowledges a fellowship from the Spanish Ministry of Education and Culture and partial support from the Spanish DGICYT contract number BFM2000-1326.

\section{References}

[1] Rafelski J and Muller B 1982 Phys. Rev. Lett. 481066

[2] Shor A 1985 Phys. Rev. Lett. 541122

[3] Ko C M and Seibert D 1994 Phys. Rev. C 492198

[4] Haglin K 1995 Nucl. Phys. A 584719

[5] Smith W and Haglin K 1998 Phys. Rev. C 571449

[6] Bando M, Kugo T and Yamawaki K 1985 Nucl. Phys. B 259493

[7] Bramon A, Grau A and Pancheri G 1995 Phys. Lett. B 345263

[8] Fujiwara T et al 1985 Prog. Theor. Phys. 73926

[9] Brown G E, Ko C M, Wu Z G and Xia L H 1991 Phys. Rev. C 431881

[10] Schnedermann E and Heinz U 1993 Phys. Rev. C 471738 